\title{
Avaliação da atividade antiviral e determinação do perfil cromatográfico de Hippeastrum glaucescens (Martius) Herbert (Amaryllidaceae)
}

\author{
Hofmann JR, A. E.1; Seben, C.'; Montanha, J.A.2; Dutilh, J.3; Sobral, M.²; \\ Henriques, A. T.2; Zuanazzi, J. A. S. ${ }^{2^{*}}$ \\ ${ }^{1}$ Faculdade de Farmácia, Universidade Regional Integrada, Campus de Erechim, RS; \\ ${ }^{2}$ Faculdade de Farmácia, Universidade Federal do Rio Grande do Sul, Porto Alegre, RS; \\ ${ }^{3}$ Instituto de Botânica, Universidade Estadual de Campinas, Campinas, SP.
}

\begin{abstract}
RESUMO: Plantas da família Amaryllidaceae são caracterizadas pela presença de alcalóides isoquinolínicos. Desde o primeiro estudo envolvendo alcalóides desta família em 1877, um grande número destas plantas tem sido analisado quimicamente. Estes compostos apresentam uma ampla variedade de atividades biológicas, tais como: antiviral, citotóxica, antitumoral e analgésica. Neste trabalho, foram avaliados o perfil cromatográfico e a potencial atividade antiviral das frações diclorometano $A$ e $B$, isoladas dos diferentes órgãos vegetais (bulbos, raízes, folhas e flores) de Hippeastrum glaucescens (Martius) Herbert, assim como dos alcalóides licorina, tazetina e pretazetina, previamente isolados desta planta. A extração dos alcalóides de $H$. glaucescens foi realizada por métodos clássicos, a partir de bulbos, raízes, folhas e flores fornecendo rendimentos totais em alcalóides de 0,53\%; 0,81\%; 0,29\% e $0,12 \%$, respectivamente. Empregando-se cromatografia em camada delgada, verificou-se que os bulbos e as raízes apresentam perfis cromatográficos semelhantes e que os alcalóides licorina, tazetina e pretazetina estão presentes em todas as partes testadas do vegetal. As frações diclorometano Ae $B$, de cada órgão vegetal, e os alcalóides isolados (licorina, tazetina e pretazetina) não inibiram a replicação do herpesvírus simples humano tipo 1 (HSV-1) cepa KOS, quando avaliados através do método de inibição do efeito citopático viral.
\end{abstract}

Unitermos: Hippeastrum glaucescens; Amaryllidaceae; alcalóides isoquinolínicos; perfil cromatográfico; atividade antiviral.

ABSTRACT: Evaluation of antiviral activity and determination of the chromatographic profile of Hippeastrum glaucescens (Martius) Herbert (Amaryllidaceae). Plants of Amaryllidaceae are characterized by isoquinoline alkaloids. Since the first study with Amaryllidaceae alkaloids in 1877, a large number of these plants have been chemically investigated. These compounds have shown a wide range of biological activities such as: antiviral, cytotoxic, antitumoral and analgesic. In this work, the dichloromethane $\left(\mathrm{CH}_{2} \mathrm{Cl}_{2}\right)$ extracts obtained from different parts of the Hippeastrum glaucescens (Martius) Herbert (bulbs, roots, leaves and flowers) and the isolated alkaloids lycorine, tazettine and pretazettine were analyzed by a chromatographic method (TLC) and tested for antiviral activity. The 
extraction of alkaloids from bulbs, roots, leaves and flowers of $H$. glaucescens was performed by classic methods and yields $0.53 \%, 0.81 \%, 0.29 \%$ and $0.12 \%$, respectively. Through TLC, bulbs and roots revealed similar chromatographic profiles and lycorine, tazettine and pretazettine were found in all the parts analyzed. The $\mathrm{CH}_{2} \mathrm{Cl}_{2}-\mathrm{A}$ and $\mathrm{CH}_{2} \mathrm{Cl}_{2}-\mathrm{B}$ extracts from each part of the plant and the isolated alkaloids (lycorine, tazettine and pretazettine) did not inhibit the HSV-1 strain KOS replication, when evaluated through the inhibition of cytophatic viral effect.

Key words: Hippeastrum glaucescens; Amaryllidaceae; isoquinoline alkaloids; chromatographic profile; antiviral activity.

\section{INTRODUÇÃO}

Os estudos com as plantas da família Amaryllidaceae despertam grande interesse, sobretudo pelas potenciais atividades farmacológicas que os seus alcalóides apresentam, como por exemplo, analgésica, citotóxica (LIN et al., 1995), antitumoral, antiviral (ANTOUN et al., 1993; DÖPKE et al., 1995; LEWIS, 1990), imunoestimulante e antifúngica (TRAM et al., 2002).

A família Amaryllidaceae compreende 14 tribos, 58 gêneros e cerca de 870 espécies (JUDD et al., 1999). Na América do Sul encontram-se aproximadamente 28 gêneros (MEEROW e SNIJMAN, 1998), sendo suas espécies conhecidas popularmente como estrela-do-norte, bastãodo-imperador (JOLY, 1966), açucena (SCHULTZ, 1990) e lírio.

Há diversos relatos do uso popular de espécies pertencentes a esta família, como por exemplo no combate ao câncer, como emético, purgativo, para o tratamento de tosses e resfriados, e externamente em contusões e contra parasitas da pele (BASTIDA et al., 1994; 1995; PETTIT et al., 1995; RAZAFIMBELO et al., 1996).

O gênero Hippeastrum pertence à tribo Hippeastreae, família Amaryllidaceae, ordem Lillifloreae e classe Monocotyledoneae (MEEROW et al., 1999; SCHULTZ, 1990). A espécie ocorre na Argentina e no Brasil, da Bahia ao Rio Grande do Sul, onde é encontrada em campos rupestres. Hofmann Jr. e colaboradores (2003) identificaram em H. glaucescens três alcalóides: licorina, tazetina e pretazetina (Figura 1).

Figura 1. Estrutura dos alcalóides identificados em Hippeastrum glaucescens (Martius) Herbert (Amaryllidaceae) $\mathrm{L}=$ licorina, $\mathrm{T}=$ tazetina e $\mathrm{P}=$ pretazetina 
Pancratistatina, um conhecido alcalóide de Amaryllidaceae, exibiu pronunciado efeito antiviral contra encefalite e o vírus da dengue (IDSO et al., 2000). Em 1992, Gabrielsen e colaboradores pesquisaram a atividade antiviral de 18 alcalóides isoquinolínicos (de origem natural e sintética) contra vírus das famílias Flaviviridae e Bunyaviridae. Neste estudo, 13 dos compostos testados, apresentaram atividade contra as flaviviroses. A seletividade dos compostos em geral foi pequena. A concentração tóxica em células não infectadas de um grande número dos alcalóides testados, foi dez vezes maior do que a concentração da atividade antiviral. Em uma revisão publicada no ano de 1991, outro trabalho relatou a atividade antiviral dos alcalóides de Amaryllidaceae em relação a flaviviroses, inclusive a febre amarela e a dengue tipo-4 (CHU e CUTLER, 1991). Extratos de Haemanthus albiflos, outra planta da família Amaryllidaceae, foram testados contra rotavírus, echovírus e vírus da poliomielite, e a atividade antiviral observada foi relacionada à presença de alcalóides como licorina e galantamina (HUSSON et al., 1991). Foi estabelecido, também, que a atividade antiviral destes alcalóides decorre geralmente de uma inibição da replicação viral e não pela inativação extracelular direta dos vírus (RENARD-NOZAKI, 1989). Para a licorina também foram comprovadas outras importantes atividades biológicas, como por exemplo, antimalárica (CAMPBELL et al., 2000), inibição da biossíntese do ácido ascórbico (LISO et al., 1984), inibição da formação de proteínas em células HeLa infectadas com poliovírus (GABRIELSEN et al., 1992) e atividade antiviral, contra vírus contendo DNA ou RNA (IEVEN et al.,1983). Estudos comparativos quanto à atividade antiproliferativa mostraram que tazetina apresentou discreta atividade, quando comparada a outros alcalóides incluindo licorina (HOHMANN et al., 2002). A pretazetina demonstrou ser ativa contra a leucemia murina de Rauscher, quando testada em camundongos, e também mostrou ser um inibidor específico da síntese de proteínas em culturas celulares (FURUSAWA et al., 1980).

Neste trabalho, estão descritos a preparação dos extratos de Hippeastrum glaucescens (Martius) Herbert, a determinação do seu perfil cromatográfico e a avaliação da atividade antiviral dos diferentes extratos e alcalóides isolados.

\section{MATERIAISE MÉTODOS}

\section{Material vegetal}

Hippeastrum glaucescens foi coletado na região nordeste do estado do Rio Grande do Sul, em dezembro de 1999. Uma exsicata encontra-se depositada no Herbário da Universidade Federal do Rio Grande do Sul / ICN (Sobral et al. 8894).

\section{Extração dos alcalóides}

A planta foi dividida em raízes $(\mathbf{R})$, bulbos $(\mathbf{B})$, folhas $(\mathbf{F O})$ e flores $(\mathbf{F L})$ e submetida à maceração estática com etanol $80 \%$ a temperatura ambiente $\left(21-24^{\circ} \mathrm{C}\right)$ por um período de $4-5$ dias. Após a maceração, seguiram-se os processos de filtração e concentração em evaporador rotatório e pressão reduzida. Os extratos concentrados foram ressuspendidos em $\mathrm{HCl} 10 \%$ e lavados inicialmente com éter de petróleo (p.e. $60-65^{\circ} \mathrm{C}$ ). Os extratos lavados foram, em seguida, extraídos com diclorometano $\left(\mathrm{CH}_{2} \mathrm{Cl}_{2}\right)$, até resultado negativo com os reativos de alcalóides Mayer, Dragendorff e Bertrand. Os extratos ácidos resultantes foram alcalinizados com $\mathrm{NH}_{4} \mathrm{OH}$ e extraídos com $\mathrm{CH}_{2} \mathrm{Cl}_{2}$ até resultado negativo com os mesmos reativos de alcalóides empregados anteriormente. A primeira extração com $\mathrm{CH}_{2} \mathrm{Cl}_{2}$ em meio ácido foi chamada de fração $\mathrm{CH}_{2} \mathrm{Cl}_{2}-\mathrm{Ae}$ a segunda, em meio alcalino, fração $\mathrm{CH}_{2} \mathrm{Cl}_{2}-\mathrm{B}$.

As frações $\mathrm{CH}_{2} \mathrm{Cl}_{2}-\mathrm{A}$ e $\mathrm{CH}_{2} \mathrm{Cl}_{2}-\mathrm{B}$ de $\mathbf{R}, \mathbf{B}, \mathrm{FO}$ e $\mathrm{FL}$ e os alcalóides isolados por Hofmann Jr. et al. (2003) (licorina, tazetina e pretazetina), foram comparados empregando-se cromatografia 
em camada delgada (CCD) sobre placas de gel de sílica e $\mathrm{CH}_{2} \mathrm{Cl}_{2}: \mathrm{MeOH}$, nas proporções 100:0, 95:5, 90:10, 85:15 e 80:20. As placas foram reveladas sob luz ultravioleta 254 e $365 \mathrm{~nm}$ e com o reagente cromogênico Dragendorff.

\section{ESTUDO DAATIVIDADEANTIVIRAL}

\section{Cultura celular}

As células VERO, fibroblastos isolados de rins de macaco verde (Cercopithecus aethiops), foram cultivadas em frascos de $75 \mathrm{~cm}^{2}$, estéreis, para cultura celular, em meio de cultura mínimo essencial de Eagle (MEM), suplementado com os antimicrobianos enrofloxacino ( $5 \mathrm{mg} / \mathrm{l})$ e fungizona ( $2 \mathrm{mg} / \mathrm{l})$, com $10 \%$ de soro bovino fetal, a $37^{\circ} \mathrm{C}$, atmosfera úmida, em uma mistura $\mathrm{ar} / \mathrm{CO}_{2}(95 \%$ / $5 \%)$.

\section{Determinação da citotoxicidade}

Para o teste de atividade antiviral foi empregado herpesvirus humano tipo 1, cepa KOS, procedente do Laboratório de Virologia Aplicada da Universidade Federal de Santa Catarina (Florianópolis). Para os testes de citotoxicidade, os extratos do vegetal e as substâncias isoladas foram solubilizados em água destilada estéril e as soluções armazenadas a $4^{\circ} \mathrm{C}$ até o momento do uso. Quando necessário, foi adicionado DMSO na concentração máxima de 0,01875 \%. A determinação da concentração máxima não tóxica (CMNT) e da concentração que provoca alteração morfológica em aproximadamente $50 \%$ do tapete celular $\left(\mathrm{CC}_{50}\right)$ foi feita em placas de microtitulação de 96 poços, com aproximadamente 40.000 células por poço, preparadas com $24 \mathrm{~h}$ de antecedância para formação do tapete celular. As soluções das substâncias ou dos extratos vegetais foram adicionadas às células, em concentrações entre 40 e 0,00977 mg/ml e as alterações morfológicas foram avaliadas após quatro dias de incubação, através da observação ao microscópio invertido em comparação ao controle celular (MONTANHA et al., 2004).

\section{Estudo da inibição do efeito citopático viral}

Assim como para a determinação da citotoxicidade, o estudo da atividade antiviral também foi feito em placas de microtitulação de 96 poços preparadas com $24 \mathrm{~h}$ de antecedência. $O$ extrato ou as substâncias puras foram adicionados à cultura celular na CMNT, em concentrações inferiores à CMNT (CMNT/2, CMNT/4 e CMNT/8), seguido da adição da suspensão viral a 100 DI. Após quatro dias de incubação, a inibição de $100 \%$ do efeito citopático viral foi avaliado em relação ao controle viral (MONTANHA et al., 2004).

\section{RESULTADOS}

A massa fresca obtida de cada órgão vegetal, bem como o rendimento das frações alcaloídicas estão apresentados na Tabela 1.

O sistema que apresentou melhor eficiência na análise por $\mathrm{CCD}$ foi $\mathrm{CH}_{2} \mathrm{Cl}_{2}: \mathrm{MeOH}(90: 10)$ em gel de sílica, tanto para as frações $\mathrm{CH}_{2} \mathrm{Cl}_{2}-\mathrm{A}$ quanto para as frações $\mathrm{CH}_{2} \mathrm{Cl}_{2}-\mathrm{B}$. Além deste, apresentaram resultados satisfatórios as proporções de 95:5 e 85:15 $\left(\mathrm{CH}_{2} \mathrm{Cl}_{2}: \mathrm{MeOH}\right)$ para a análise das manchas mais apolares e mais polares, respectivamente. 
Tabela 1. Rendimentos do processo de extração dos alcalóides de Hippeastrum glaucescens (Martius) Herbert (Amaryllidaceae) em relação à quantidade de cada material vegetal utilizado (bulbos, raízes, folhas e flores).

\begin{tabular}{|c|c|c|c|c|c|}
\hline & MV (g) & $\begin{array}{c}\text { RA } \\
(\%, \mathbf{m} / \mathbf{m})\end{array}$ & $\begin{array}{c}\text { RB } \\
(\%, \mathbf{m} / \mathbf{m})\end{array}$ & $\begin{array}{c}\text { RT } \\
(\%, \mathbf{m} / \mathbf{m})\end{array}$ \\
\hline BULBOS (B) & 3.150 & 0,04 & 0,49 & 0,53 \\
\hline RAízES (R) & 575 & 0,07 & 0,74 & 0,81 \\
\hline FOLHAS (FO) & 1.565 & 0,19 & 0,10 & 0,29 \\
\hline FLORES (FL) & 147 & 0,05 & 0,08 & 0,12 \\
\hline
\end{tabular}

$\mathrm{MV}=$ massa em gramas do vegetal fresco; $\mathrm{RA}=$ rendimento da fração $\mathrm{CH}_{2} \mathrm{Cl}_{2}-\mathrm{A}$; $\mathrm{RB}$ = rendimento da fração $\mathrm{CH}_{2} \mathrm{Cl}_{2}-\mathrm{B} ; \mathrm{RT}$ = rendimento total em alcalóides.

O número de manchas observadas nas frações $\mathrm{CH}_{2} \mathrm{Cl}_{2}-\mathrm{A}$ é menor que nas frações $\mathrm{CH}_{2} \mathrm{Cl}_{2}$ $\mathrm{B}$, em todas as partes do vegetal (R, B, FO e FL). As frações $\mathrm{CH}_{2} \mathrm{Cl}_{2}-\mathrm{A}_{\text {e }} \mathrm{CH}_{2} \mathrm{Cl}_{2}-\mathrm{B}$ de $\mathbf{F L}$ exibiram um número menor de manchas quando comparadas às demais. As frações $\mathrm{CH}_{2} \mathrm{Cl}_{2}-\mathrm{B}$ de $\mathbf{B}$ e $\mathbf{R}$ apresentaram perfis cromatográficos muito semelhantes, o que também pode ser observado com as frações $\mathrm{CH}_{2} \mathrm{Cl}_{2}$-A de $\mathbf{B}, \mathbf{R}$ e FO. Todavia, o cromatograma de $\mathbf{F O}$ apresentou maior número de manchas do que os de $\mathbf{B}$ e $\mathbf{R}$. As frações $\mathrm{CH}_{2} \mathrm{Cl}_{2}-\mathrm{Ae} \mathrm{CH}_{2} \mathrm{Cl}_{2}-\mathrm{B}$ apresentaram algumas manchas de $R f$ semelhantes, revelados sob luz UV $254 \mathrm{~nm}$. Contudo, quando observado sob luz UV 365 $\mathrm{nm}$, verificou-se que as mesmas apresentaram fluorescências diferentes.

No eluente $\mathrm{CH}_{2} \mathrm{Cl}_{2}: \mathrm{MeOH}(90: 10)$, o alcalóide licorina apresentou $R f$ próximo a 0,40, com fluorescência amarela-avermelhada, quando revelado sob luz UV $365 \mathrm{~nm}$, e fluorescência levemente azulada sob luz UV $254 \mathrm{~nm}$, tendo reagido positivamente com o reativo de Dragendorff. Este alcalóide foi identificado em todas as frações $\mathrm{CH}_{2} \mathrm{Cl}_{2}-\mathrm{B}(\mathbf{R}, \mathbf{B}, \mathbf{F O}$ e $\mathbf{F L})$, não estando presente nas frações $\mathrm{CH}_{2} \mathrm{Cl}_{2}-\mathrm{A}$.

O alcalóide tazetina apresentou Rfpróximo a 0,60 no eluente $90: 10\left(\mathrm{CH}_{2} \mathrm{Cl}_{2}: \mathrm{MeOH}\right)$, tendo sido visualizado com extinção da fluorescência da placa sob luz UV 254 nm e fluorescência violeta muito fraca quando revelado sob luz UV $365 \mathrm{~nm}$. Após revelação com o reativo de Dragendorff, foi visualizado nas frações $\mathrm{CH}_{2} \mathrm{Cl}_{2}-\mathrm{B}$ de $\mathbf{B}$ e $\mathbf{R}$. Nas frações de $\mathbf{F O}$ e $\mathbf{F L}$, as manchas estão muito próximas à outra mancha de $R f$ semelhante. Da mesma forma que a licorina, a tazetina não foi identificada nas frações $\mathrm{CH}_{2} \mathrm{Cl}_{2}-\mathrm{A}$.

O alcalóide pretazetina, quando analisado no eluente $90: 10\left(\mathrm{CH}_{2} \mathrm{Cl}_{2}: \mathrm{MeOH}\right)$, foi detectado sob luz UV 365 nm, apresentando fluorescência azul fraca e, sob luz UV 254 nm, com extinção da fluorescência, em um $R f$ próximo a 0,20. Apresentou reação positiva com o reativo de Dragendorff, estando presente em todas as frações $\mathrm{CH}_{2} \mathrm{Cl}_{2}-\mathbf{B}$ ( $\mathbf{R}, \mathbf{B}$, FO e FL), porém não foi encontrado nas frações $\mathrm{CH}_{2} \mathrm{Cl}_{2}-\mathrm{A}$.

Nenhum dos extratos analisados, nem os alcalóides isolados do vegetal (licorina, tazetina e pretazetina) foram capazes de inibir o efeito citopático do HSV-1 cepa KOS, em células Vero, empregando a metodologia descrita. 


\section{DISCUSSÃO}

A taxonomia da família Amaryllidaceae é bastante complexa devido à falta de parâmetros morfológicos estáveis e constantes, que caracterizam os diferentes grupos, fornecendo uma clara delimitação entre eles. Adicionalmente, um outro complicador é a possibilidade de formação de híbridos. Assim, a identificação do conteúdo em alcalóides, marcadores químicos para a família, pode ser uma ferramenta bastante útil, contribuindo para a compreensão das relações filogenéticas entre os gêneros e as espécies.

Foi observado, no processo de extração da planta, que os reativos de Mayer e Dragendorff foram menos sensíveis que o reativo de Bertrand para a classe de compostos alcaloídicos presentes nesta espécie.

Durante o processo de obtenção dos alcalóides totais, verificou-se que a etapa de lavagem do extrato ácido com diclorometano apresentou reação positiva aos reativos de precipitação de alcalóides. Esta fração, que geralmente não contém alcalóides, foi chamada $\mathrm{CH}_{2} \mathrm{Cl}_{2}-\mathrm{A}$. Este fato pode estar relacionado à presença de substâncias com menor probabilidade de formação de sais conjugados no pH utilizado (entre 2 e 3), permanecendo assim na forma de base livre, sendo extraídas com solvente orgânico $\left(\mathrm{CH}_{2} \mathrm{Cl}_{2}\right)$. A fração, obtida após alcalinização do extrato aquoso e extração com diclorometano, foi chamada de $\mathrm{CH}_{2} \mathrm{Cl}_{2}-\mathrm{B}$, sendo mais rica em alcalóides para todos

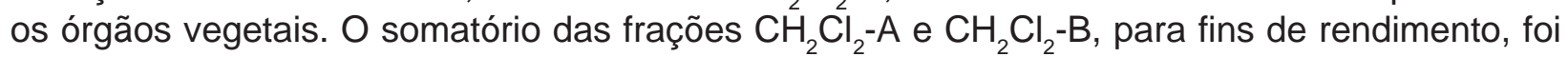
considerado como o teor total de alcalóides na planta.

A partir das análises cromatográficas realizadas (CCD), foi verificado que as frações de bulbos e raízes apresentaram comportamentos cromatográficos muito semelhantes, diferenciandose das mesmas frações obtidas de flores e folhas. Inicialmente, este dado indica que as folhas e flores do vegetal devem ser analisadas separadamente e os bulbos e raízes não necessitam ser separados.

Através da análise em CCD foi observado que os alcalóides licorina, tazetina e pretazetina estão presentes em todos os órgãos do vegetal.

Apesar da avaliação de atividade antiviral não ter apresentado, neste modelo experimental, efeito positivo e haja visto o grande número de efeitos descritos na literatura para estes alcalóides, sugere-se que outros modelos devem ser testados.

\section{AGRADECIMENTOS}

AFAPERGS, CAPES e CNPQ pelo aporte financeiro.

\section{REFERÊNCIAS}

ANTOUN, M. D.; MENDOZA, N. T.; RÍOS, Y. R.; PROCTOR, G. R.; WICKRAMARATNE, D. B. M.; PEZZUTO, J. M.; KINGHORN, A. D. Cytotoxicity of Hymenocallis expansa alkaloids. Journal of Natural Products, v. 56, n. 8, p. 1423-1425, 1993.

BASTIDA, J.; BERGOÑÓN, S.; VILADOMAT, F.; CODINA, C. Alkaloids from Narcissus primigenius. Phytochemistry, v. 60, p. 95-96, 1994.

BASTIDA, J.; CODINA, C.; PEETERS, P.; RUBIRALTA, M.; OROZCO, M.; LUQUE, F. J.; CHHABRA, S. C. Alkaloids from Crinum kirkii. Phytochemistry, v. 40, p. 1291-1293, 1995.

CAMPBELL, W. E.; NAIR, J. J.; GAMMON, D. W.; CODINA, C.; BASTIDA, J.; VILADOMAT, F.; SMITH, P. J.; ALBRECHT, C. F. Bioactive alkaloids from Brunsvigia radulosa. Phytochemistry, v. 53, p. 587-591, 2000.

Rev. Bras. Farmacogn., V. 14, n. 1, jan.-jun. 2004. 
CHU, C. K.; CUTLER, H. G. Natural products as antiviral agents. New York: Plenum, p. 121-135, 1991.

DÖPKE, W.; PHAM, L. H.; GRÜNDEMANN, E.; BARTOSZEK, M.; FLATAU, S. Alkaloids from Hippeastrum equestre; Part I: phamine, a new phenanthridone alkaloid. Planta Medica, v. 61, p. 564-566, 1995.

FURUSAWA, E.; IRIE, H.; COMBS, D.; WILDMAN, W. Therapeutic activity of pretazettine on Rauscher leukemia: Comparison with the related Amaryllidaceae alkaloids. Chemotherapy, v. 26, p. 36-45, 1980.

GABRIELSEN, B.; MONATH, T.; HUGGINS, J. W.; KEFAUVER, D. Antiviral (RNA) activity of selected Amaryllidaceae isoquinoline constituents and synthesis of related substances. Journal of Natural Products, $v$. 55, n. 11, p. 1569-1581, 1992.

HOFMANN Jr, A. E.; SEBBEN C.; SOBRAL, M. E.; DUTILH, J.; HENRIQUES, A. T.; ZUANAZZI, J. A. S. Alkaloids of Hippeastrum glaucescens. Biochemical Systematics and Ecology, v. 31, p. 1455-1456, 2003.

HOHMANN, J.; FORGO, P.; MOLNÁR, J.; WOLFARD, K.; MOLNÁR, A.; THALHAMMER, T.; MÁTHÉ, I.; SHARPLES, D. Antiproliferative Amaryllidaceae alkaloids isolated from the bulbs of Sprekelia formosissima and Hymenocallis x festalis. Planta Medica, v. 68, p. 452-454, 2002.

HUSSON, G. P.; VILAGINES, P.; SARRETE, B.; VILAGINES, R. Effet antiviral d'un extrait de bulbe d'Amaryllidaceae sur trois entérovirus et un réoviride. Plantes Médicinales et Phytothérapie, n. 2-3, p. 89-99, 1991.

IDSO, S. B.; KIMBALL, B. A.; PETTIT III, G. R.; GARNER, L. C.; PETTIT, G. R.; BACKHAUS, R. A. Effects of atmospheric $\mathrm{CO}_{2}$ enrichment on the growth and development of Hymenocallis littoralis (Amaryllidaceae) and the concentrations of several antineoplastic and antiviral constituents of its bulbs. American Journal of Botany, v. 87-6, p. 769-773, 2000.

IEVEN, M.; VAN DEN BERGHE, D. A.; VLIETINCK, A. J. Plant antiviral agents IV. Influence of lycorine on growth pattern of three animal viruses. Planta Medica, v. 49, p.109-114, 1983.

JOLY, A. Botânica: Introdução a taxonomia. São Paulo: Editora da Universidade de São Paulo, 1966.

JUDD, W. S.; CAMPBELL, C. S.; KELLOGG, E. A.; STEVENS, P. F. Plant Systematics: A phylogenetic approch. New York: Sunderland \& Sinauer, p. 464, 1999.

LEWIS, J. R. Amaryllidaceae alkaloids. Natural Product Reports, v. 7, p. 549-556, 1990.

LIN, L.; HU, S.; CHAI, H.; PENGSUPARP, T.; PEZZUTO, J. M.; CORDELL G. A., RUANGRUNGSI N. Lycorine alkaloids from Hymenocallis littoralis. Phytochemistry, v. 40, p. 1295-1298, 1995.

LISO, R.; CALABRESE, G.; BITONTI, M. B.; ARRIGONI, O. Relationship between ascorbic acid and cell division. Experimental Cell Research, v. 150, p. 314-320, 1984.

MONTANHA, J. A.; MOELLERKE, P.; BORDIGNON, S. A. L. J.; SCHENKEL, E. P.; ROEHE, P. M. Antiviral activity of Brazilian plant extracts. Acta Farmacéutica Bonaerense, 2004, aceito para publicação.

MEEROW, A. W.; SNIJMAN, D. A. In: KUBITZKI K. (Ed). The Familes and Genera of Vascular Plants. Berlin: Springer, 1998. v. 3.

MEEROW, A. W.; FAY, M. F.; GUY, C. L.; LI, Q.; ZAMAN, F. Q.; CHASE, M. W. Systematics of Amaryllidaceae based on cladistic analysis of plastid $r b c L$ and $t r n L-F$ sequence data. American Journal of Botany, v. 86, p. 1325-1345, 1999.

PETTIT, G. R.; PETTIT III, G. R.; BACKHAUS, R. A.; BOETTNER, F. E. Antineoplasic agents, 294. Variations in the formation of pancratistatin and related isocarbostyrils in Hymenocallis littoralis. Journal of Natural Products, v. 58, p. 37-43, 1995.

RAZAFIMBELO, J.; ANDRIANTSINFERANA, M.; BAUDOUIN, G.; TILLEQUIN, F. Alkaloids from Crinum firmifolium var. hygrophilum. Phytochemistry, v. 41, p. 323-326, 1996.

RENARD-NOZAKI, J.; KIM, T.; IMAKURA, Y.; KIHARA, M.; KOBAYASHI, S. Effect of alkaloids isolated from Amaryllidaceae on Herpex Simplex Virus. Research in Virology, v.140, p. 115-128, 1989. 
SCHULTZ, A. Introdução a Botânica Sistemática. 6. ed. Porto Alegre: Editora da Universidade Federal de Rio Grande do Sul, 1990. v. 2.

TRAM, N. T. M.; TITORENKOVA, T. V., BANKOVA, V. S.; HANDJIEVA, N.V.; POPOV, S. S. Crinum L. (Amaryllidaceae). Fitoterapia, v. 73, p. 183-208, 2002. 


\title{
ERRATA
}

Revista Brasileira de Farmacognosia, V. 14, n. 1, p.7-14, 2004.

ISSN 0102-695X

\section{Avaliação da atividade antiviral e determinação do perfil cromatográfico de Hippeastrum glaucescens (Martius) Herbert (Amaryllidaceae)}

\author{
Hofmann Jr., A. E..; Seben, C.'; Montanha, J.A.2; Dutilh, J..; Sobral, M.; \\ Henriques, A. T. ${ }^{2}$ Z Zuanazzi, J. A. S. ${ }^{2 *}$ \\ ${ }^{1}$ Faculdade de Farmácia, Universidade Regional Integrada, Campus de Erechim, RS; \\ 2 Faculdade de Farmácia, Universidade Federal do Rio Grande do Sul, Porto Alegre, RS; \\ ${ }^{3}$ Instituto de Botânica, Universidade Estadual de Campinas, Campinas, SP.
}

O gênero Hippeastrum pertence à tribo Hippeastreae, família Amaryllidaceae, ordem Lillifloreae e classe Monocotyledoneae (MEEROW et al., 1999; SCHULTZ, 1990). A espécie ocorre na Argentina e no Brasil, da Bahia ao Rio Grande do Sul, onde é encontrada em campos rupestres. Hofmann Jr. e colaboradores (2003) identificaram em H. glaucescens três alcalóides: licorina, tazetina e pretazetina (Figura 1).<smiles>OC1C=C2CCN3Cc4cc5c(cc4C(C1O)C23)OCO5</smiles>

$\mathbf{L}$<smiles>COC1C=CC2(OCc3cc4c(cc32)OCO4)C(O)(O)C1</smiles>

$\mathbf{T}$<smiles>COC1C=CC23c4cc5c(cc4C(O)OC2CN(C)C3C1)OCO5</smiles>

Figura 1. Estrutura dos alcalóides identificados em Hippeastrum glaucescens (Martius) Herbert (Amaryllidaceae): $\mathrm{L}=$ licorina, $\mathrm{T}=$ tazetina $\mathrm{e} \mathrm{P}=$ pretazetina. 
O arquivo disponível sofreu correções conforme ERRATA publicada no Volume 14 Número 2 da revista. 